F. S. Cater, Department of Mathematics, Portland State University, Portland, Oregon 97207, USA

\title{
A CHANGE OF VARIABLES FORMULA FOR DARBOUX INTEGRALS
}

\begin{abstract}
We offer an inequality involving upper and lower Darboux integrals of bounded real functions which implies a number of variations of the change of variables formula for Riemann integrals.
\end{abstract}

Throughout this paper $D g$ denotes one of the four Dini derivates of a continuous real valued function $g$ on a compact interval $[a, b]$ (the same Dini derivate at all $x$ ). Let $g(a) \leq g(b)$ and let $D g$ be bounded on $[a, b]$. Let $f$ be a bounded function on $g[a, b]$ such that for almost every $t \in[a, b]$, one or both of the functions $f(g(\cdot))$ or $D g(\cdot)$ is continuous at $t$.

We offer the following change of variables formula for Darboux integrals.

Theorem 1. For upper and lower Darboux integrals we have the inequality:

$$
\begin{aligned}
\overline{\int_{a}^{b}} f(g(t)) D g(t) d t & \geq \overline{\int_{g(a)}^{g(b)}} f(x) d x \\
& \geq \underline{\int_{g(a)}^{g(b)}} f(x) d x \geq \underline{\int_{a}^{b}} f(g(t)) D g(t) d t .
\end{aligned}
$$

Moreover, if $f(g(t)) D g(t)$ is R-integrable on $[a, b]$, then $f(x)$ is $R$-integrable on $[g(a), g(b)]$ and

$$
\int_{a}^{b} f(g(t)) D g(t) d t=\int_{g(a)}^{g(b)} f(x) d x .
$$

The inspiration for this work was references $[\mathrm{H}]$ and $[\mathrm{T}]$, which provided essentially the following proposition.

Key Words: Darboux integral, Riemann integral.

Mathematical Reviews subject classification: 26A39, 26A42.

Received by the editors March 1, 1998 
Proposition 1. In Theorem 1, let Dg be continuous almost everywhere on $[a, b]$ and let $g$ be nondecreasing. Then we have

$$
\begin{aligned}
\overline{\int_{a}^{b}} f(g(t)) D g(t) d t & =\overline{\int_{g(a)}^{g(b)}} f(x) d x \\
& \geq \underline{\int_{g(a)}^{g(b)}} f(x) d x=\underline{\int_{a}^{b}} f(g(t)) D g(t) d t .
\end{aligned}
$$

The continuity hypothesis in this paper is satisfied, for example, when $f$ is everywhere continuous; likewise it is satisfied when $D g$ is continuous almost everywhere on $[a, b]$ as in Proposition 1. The hypothesis of Theorem 1 subsumes both of these cases.

Theorem 1 has immediate applications to Riemann integration. We offer some of these.

Corollary 1. Let $k$ be a real valued function on $[a, b]$ with a bounded difference quotient, and $k(a) \leq k(b)$. Let $h$ be a bounded function on $k[a, b]$. If two of the three functions $h(k(t)) D k(t), h(k(t)), D k(t)$ are $R$-integrable on $[a, b]$, then $h$ is R-integrable on $[k(a), k(b)], h(k(t)) D k(t)$ is $R$-integrable on $[a, b]$, and

$$
\int_{a}^{b} h(k(t)) D k(t) d t=\int_{k(a)}^{k(b)} h(x) d x .
$$

To prove this from Theorem 1 , recall that a bounded function is $R$-integrable on $[a, b]$ if and only if it is continuous almost everywhere on $[a, b]$. Thus in particular, the product of two $R$-integrable functions is $R$-integrable on $[a, b]$.

Corollary 2. In Theorem 1 let $f(g(t)) D g(t)$ be R-integrable on $[a, b]$. Then $f$ is $R$-integrable on the interval $g[a, b]$.

To prove this apply Theorem 1 on the intervals $[a, s]$ and $[r, b]$ where $g$ takes its maximum at $s$ and its minimum at $r$.

Corollary 3. In Theorem 1 let $f$ be R-integrable on $g[a, b]$ and let $D g$ be $R$-integrable on $[a, b]$. Then $f(g(t)) D g(t)$ is $R$-integrable on $[a, b]$ and

$$
\int_{a}^{b} f(g(t)) D g(t) d t=\int_{g(a)}^{g(b)} f(x) d x .
$$

(See also $[V])$. 
PROOF. In view of Theorem 1 , it suffices to prove that $m(X)=0$ where

$$
X=\{x: f(g(t)) D g(t) \text { is discontinuous at } x\} \text {. }
$$

Put

$$
X_{1}=\{x \in X: D g \text { is continuous at } x\} .
$$

Then $m\left(X \backslash X_{1}\right)=0$ because $D g$ is $R$-integrable on $[a, b]$. Also $D g(x) \neq 0$ for each $x \in X_{1}$ because $D g$ is continuous at $x$ and $f$ is bounded. Moreover, $f$ is discontinuous at $g(x)$ for each $x \in X_{1}$. Now each $x \in X_{1}$ lies in an open interval $I_{x}$ with $D g$ bounded away from 0 on $I_{x}$. Thus $g$ has an absolutely continuous inverse $g^{-1}$ on $g\left(I_{x}\right)$. But $m\left(g\left(X_{1}\right)\right)=0$ because $f$ is $R$-integrable. Hence

$$
m\left(I_{x} \cap X_{1}\right) \leq m\left(g^{-1}\left(g\left(I_{x} \cap X_{1}\right)\right)\right)=0 .
$$

Thus $X_{1}$ is the union of countably many subsets of measure 0 , so $m\left(X_{1}\right)=$ $m(X)=0$.

Corollary 4. Let (**) hold in Theorem 1. Then $f(g(t)) D g(t)$ is R-integrable on any interval $[u, v]$ for which $g(u)=g(v)$.

We defer the proof of Corollary 4 until we prove Theorem 1. Finally we show by example that the equations in $(* *)$ need not hold in Theorem 1.

Our techniques will be quite different from those used for change of variables for the Lebesgue and other integrals. Compare with the work found in references $[\mathrm{F}],[\mathrm{G} 1],[\mathrm{G} 2],[\mathrm{K}],[\mathrm{V}]$, for example.

We turn now to some definitions we will use. We say that a finite increasing sequence $a=x_{0}<x_{1}<\ldots<x_{n}=b$ is a partition of the interval $[a, b]$. The $x_{i}$ are called the dividing points of this partition. By the norm of the partition we mean $\max _{i=1}^{n}\left(x_{i}-x_{i-1}\right)$. By the inherited partition of the subinterval $[u, v]$ of $[a, b]$ we mean the partition whose dividing points are $u, v$ and all the $x_{i}$ between $u$ and $v$. We say that another partition of $[a, b], a=y_{0}<y_{1}<\ldots<$ $y_{m}=b$ is a refinement of this one if for each $x_{i}$ there is a $y_{j}$ with $y_{j}=x_{i}$.

We say that the partition $a=x_{0}<x_{1}<\ldots<x_{n}=b$ is a special partition of $[a, b]$ if there is a finite increasing sequence of integers $0=n_{0}<n_{1}<\ldots<$ $n_{k}=n$ such that for each index $j=0,1, \ldots, k-1$ either $g\left(x_{n_{j}}\right)=g\left(x_{n_{j+1}}\right)$, or $n_{j+1}=n_{j}+1$ and $g\left(x_{n_{j}}\right)<g\left(x_{n_{j+1}}\right)$ and $g\left(x_{n_{j}}, x_{n_{j+1}}\right)=\left(g\left(x_{n_{j}}\right), g\left(x_{n_{j+1}}\right)\right)$.

By a Riemann sum for $f(g(t)) D g(t)$ on the partition $a=x_{0}<x_{1}<\ldots<$ $x_{n}=b$ we mean a sum of the form

$$
\sum_{i=1}^{n} f\left(g\left(t_{i}\right)\right) D g\left(t_{i}\right)\left(x_{i}-x_{i-1}\right)
$$


where $t_{i}$ is any point in the interval $\left[x_{i-1}, x_{i}\right]$. If $f\left(g\left(t_{i}\right)\right) D g\left(t_{i}\right)$ is replaced by

$$
\sup \left\{f(g(t)) D g(t): t \in\left[x_{i-1}, x_{i}\right]\right\}
$$

the sum is called an upper sum. Likewise when $f\left(g\left(t_{i}\right)\right) D g\left(t_{i}\right)$ is replaced by

$$
\inf \left\{f(g(t)) D g(t): t \in\left[x_{i-1}, x_{i}\right]\right\}
$$

the sum is called a lower sum. Similar definitions are given for $f$ on partitions of the interval $[g(a), g(b)]$.

By a mixed sum on the partition $a=x_{0}<x_{1}<\ldots<x_{n}=b$ we mean a sum of the form $\sum_{i=1}^{n} f\left(g\left(s_{i}\right)\right) D g\left(t_{i}\right)\left(x_{i}-x_{i-1}\right)$ where $s_{i}$ and $t_{i}$ are in $\left[x_{i-1}, x_{i}\right]$. Thus a Riemann sum for $f(g(t)) D g(t)$ is a particular kind of mixed sum.

Special partitions and mixed sums are ad hoc definitions in this paper.

Lemma 1. Let $a=x_{0}<x_{1}<\ldots<x_{n}=b$ be a partition of $[a, b]$. Then it has a refinement that is a special partition of $[a, b]$.

Proof. Assume $g(a)<g(b)$; otherwise $g(a)=g(b)$ and the given partition is a special partition.

Let $y$ be the least number in the set $[a, b] \cap g^{-1}(g(b))$, let $z$ be the greatest number in the set $[a, b] \cap g^{-1}(g(a))$, and let $w$ be the greatest number in the set $[a, y] \cap g^{-1}(g(a))$.

We use induction on $n$. If $n=1$, the dividing points in the special partition are the distinct points among $a, b, y$ and $w$. Note that $g(w, y)=(g(w), g(y))$ if $w<y, g(a)=g(w)$ if $a<w$ and $g(y)=g(b)$ if $y<b$.

Assume $n \geq 2$ and the conclusion holds for partitions of any subinterval of $[a, b]$ with fewer than $n+1$ dividing points. We have three cases.

CASE 1. $g\left(x_{n-1}\right) \geq g(b)$. Then $y \leq x_{n-1}$. Use $g(b)=g(y)$ and the induction hypothesis on the inherited partition of the interval $[a, y]$.

CASE 2. $g\left(x_{n-1}\right) \leq g(a)$. Then $x_{n-1} \leq z$. Use $g(a)=g(z)$ and the induction hypothesis on the inherited partition of the interval $[z, b]$.

CASE 3. $g(a)<g\left(x_{n-1}\right)<g(b)$. Use the induction hypothesis on the respective inherited partitions of the intervals $\left[a, x_{n-1}\right]$ and $\left[x_{n-1}, b\right]$.

This covers all cases, and the induction is proved.

Lemma 2. Let $a=x_{0}<x_{1}<\ldots<x_{n}=b$ be a partition of $[a, b]$. Let $\delta>0$. Then there is a refinement of this partition that is a special partition of $[a, b]$ of norm $<\delta$.

Proof. Take a refinement of norm $<\delta$ and apply Lemma 1 to it. 
Lemma 3. Let $a=x_{0}<x_{1}<\ldots<x_{n}=b$ be any partition of $[a, b]$, let $g(a)=g(b)$, and $\epsilon>0$. Then there is a refinement of this partition with a mixed sum on it $>-\epsilon(<\epsilon)$.

Proof. The proof follows by induction on $n$. Let $n=1$. Choose any $x \in[a, b]$. We use $g(b)-g(a)=0$ and Dini's Theorem [S] to select $t \in[a, b]$ such that

$$
f(g(x)) D g(t)(b-a)>-\epsilon
$$

as follows. For $f(g(x))>0$ choose $t$ so that

$$
D g(t)>(g(b)-g(a))(b-a)^{-1}-\epsilon f(g(x))^{-1}(b-a)^{-1}
$$

and multiply by the positive number $f(g(x))(b-a)$; for $f(g(x))<0$ choose $t$ so that

$$
D g(t)<(g(b)-g(a))(b-a)^{-1}-\epsilon f(g(x))^{-1}(b-a)^{-1}
$$

and multiply by the negative number $f(g(x))(b-a)$; for $f(g(x))=0$, any $t$ will do.

Now assume that $n \geq 2$ and the conclusion holds when $[a, b]$ is replaced by any subinterval, $\epsilon$ is replaced by any positive number, and the partition has fewer than $n+1$ dividing points.

CASE 1. $g\left(x_{1}\right)=g(a)$ or $g\left(x_{n-1}\right)=g(b)$. Use the induction hypothesis on the respective inherited partitions of the intervals $\left[a, x_{1}\right]$ and $\left[x_{1}, b\right]$, or $\left[a, x_{n-1}\right]$ and $\left[x_{n-1}, b\right]$.

CASE 2. $g\left(x_{1}\right)<g(a)<g\left(x_{n-1}\right)$. Let $q \in\left(x_{1}, x_{n-1}\right)$ such that $g(q)=$ $g(a)$. Use the induction hypothesis on the respective inherited partitions of the intervals $[a, q]$ and $[q, b]$.

CASE 3. $g\left(x_{1}\right)>g(a)>g\left(x_{n-1}\right)$. Analogous to Case 2 .

CASE 4. $g\left(x_{1}\right) \geq g\left(x_{n-1}\right)>g(a)$. Let $u$ be the smallest number in the set $[a, b] \cap g^{-1}\left(g\left(x_{n-1}\right)\right)$. Then $u \leq x_{1}$, and the Intermediate Value Theorem may be used to select $v_{1} \in[a, u]$ and $v_{2} \in\left[x_{n-1}, b\right]$ such that $g\left(v_{1}\right)=g\left(v_{2}\right)$. As in the proof for $n=1$, use Dini's Theorem [S] to choose $r \in[a, u]$ and $s \in\left[x_{n-1}, b\right]$ such that

$$
\begin{gathered}
f\left(g\left(v_{1}\right)\right) D g(r)(u-a)>f\left(g\left(v_{1}\right)\right)(g(u)-g(a))-\frac{1}{4} \epsilon, \\
f\left(g\left(v_{2}\right)\right) D g(s)\left(b-x_{n-1}\right)>f\left(g\left(v_{2}\right)\right)\left(g(b)-g\left(x_{n-1}\right)\right)-\frac{1}{4} \epsilon .
\end{gathered}
$$

Recall that $g(a)=g(b), g(u)=g\left(x_{n-1}\right), g\left(v_{1}\right)=g\left(v_{2}\right)$, and add (1) and (2) to obtain

$$
f\left(g\left(v_{1}\right)\right) D g(r)(u-a)+f\left(g\left(v_{2}\right)\right) D g(s)\left(b-x_{n-1}\right)>-\frac{1}{2} \epsilon .
$$


Apply the induction hypothesis to the inherited partition of the subinterval $\left[u, x_{n-1}\right]$ (with $\frac{1}{2} \epsilon$ ), and add the inequality obtained to (3).

CASE 5. $g\left(x_{n-1}\right) \geq g\left(x_{1}\right)>g(a)$. Let $u$ be the largest number in the set $[a, b] \cap g^{-1}\left(g\left(x_{1}\right)\right)$. Then $u \geq x_{n-1}$. Argue as in Case 4 and use the subintervals $\left[a, x_{1}\right],\left[x_{1}, u\right]$ and $[u, b]$.

CASE 6. $g\left(x_{1}\right)<g(a)$ and $g\left(x_{n-1}\right)<g(a)$. This is analogous to Cases 4 and 5 , so we leave it.

This covers all the cases to prove the induction for the first inequality (with $>-\epsilon$ ). The second inequality (with $<\epsilon$ ) is proved analogously. We leave the rest.

Lemma 4. Let $\epsilon>0$ and $\delta>0$. Then there is a special partition of $[a, b]$ with norm $<\delta$ and a mixed sum on it

$$
>\overline{\int_{g(a)}^{g(b)}} f(x) d x-\epsilon .
$$

Proof. We can dismiss the case in which $g(a)=g(b)$; for here we just apply Lemma 3 to any partition of $[a, b]$ of norm $<\delta$. So assume $g(a)<g(b)$.

Let $a=x_{0}<x_{1}<\ldots<x_{n}=b$ be a special partition of $[a, b]$ of norm $<\delta$ (Lemma 2). Say $0=n_{0}<n_{1}<\ldots<n_{k}=n$ such that for any $j=0,1, \ldots, k-1$, either $g\left(x_{n_{j}}\right)=g\left(x_{n_{j+1}}\right)$ or $g\left(x_{n_{j}}\right)<g\left(x_{n_{j+1}}\right)$ and $g\left(x_{n_{j}}, x_{n_{j+1}}\right)=\left(g\left(x_{n_{j}}\right), g\left(x_{n_{j+1}}\right)\right)$ and $n_{j+1}=n_{j}+1$. For any such $j$ we consider the two possibilities.

CASE 1. $g\left(x_{n_{j}}\right)=g\left(x_{n_{j+1}}\right)$. Use Lemma 3 to obtain a refinement of the inherited partition of the interval $\left[x_{n_{j}}, x_{n_{j+1}}\right]$ and a mixed sum on it

$$
>\overline{\int_{g\left(x_{n_{j}}\right)}^{g\left(x_{n_{j+1}}\right)}} f(x) d x-\epsilon(2 k)^{-1} .
$$

CASE 2. $g\left(x_{n_{j}}, x_{n_{j+1}}\right)=\left(g\left(x_{n_{j}}\right), g\left(x_{n_{j+1}}\right)\right)$. Let $M$ denote the sup of $f$ on the interval $\left[g\left(x_{n_{j}}\right), g\left(x_{n_{j+1}}\right)\right]$. We use Dini's Theorem [S] to find a $t \in\left[x_{n_{j}}, x_{n_{j+1}}\right]$ such that

$$
M D g(t)\left(x_{n_{j+1}}-x_{n_{j}}\right)>M\left(g\left(x_{n_{j+1}}\right)-g\left(x_{n_{j}}\right)\right)-\epsilon(2 k)^{-1}
$$

as follows. Choose $t$ so that

$D g(t)>\left(g\left(x_{n_{j+1}}\right)-g\left(x_{n_{j}}\right)\right)\left(x_{n_{j+1}}-x_{n_{j}}\right)^{-1}-\epsilon(2 k)^{-1} M^{-1}\left(x_{n_{j+1}}-x_{n_{j}}\right)^{-1}$ 
an multiply by $M\left(x_{n_{j+1}}-x_{n_{j}}\right)$ if $M$ is positive; choose $t$ so that

$D g(t)<\left(g\left(x_{n_{j+1}}\right)-g\left(x_{n_{j}}\right)\right)\left(x_{n_{j+1}}-x_{n_{j}}\right)^{-1}-\epsilon(2 k)^{-1} M^{-1}\left(x_{n_{j+1}}-x_{n_{j}}\right)^{-1}$

an multiply by $M\left(x_{n_{j+1}}-x_{n_{j}}\right)$ if $M$ is negative; choose any $t$ if $M=0$. Then we choose $s \in\left[x_{n_{j}}, x_{n_{j+1}}\right]$ so that

$$
\begin{gathered}
f(g(s)) D g(t)\left(x_{n_{j+1}}-x_{n_{j}}\right)>M\left(g\left(x_{n_{j+1}}\right)-g\left(x_{n_{j}}\right)\right)-\epsilon(2 k)^{-1} \\
\geq \overline{\int_{g\left(x_{n_{j}}\right)}^{g\left(x_{n_{j+1}}\right)}} f(x) d x-\epsilon(2 k)^{-1} .
\end{gathered}
$$

From Cases 1 and 2 we easily see that there is a refinement of $a=x_{0}<$ $x_{1}<\ldots<x_{n}=b$ and a mixed sum on it

$$
\geq \sum_{j=0}^{k-1} \overline{\int_{g\left(x_{n_{j}}\right)}^{g\left(x_{n_{j+1}}\right)}} f(x) d x-\epsilon=\overline{\int_{g(a)}^{g(b)}} f(x) d x-\epsilon .
$$

So far we have not used the continuity hypothesis on $f$ and $D g$. Now we use this hypothesis to change from mixed sums to Riemann sums and complete the proof of Theorem 1 .

Lemma 5. Let $\epsilon>0$. Then there is a $\delta>0$ such that for any partition $a=x_{0}<x_{1}<\ldots<x_{n}=b$ of $[a, b]$ with norm $<\delta$ and for any mixed sum $W$ on this partition, there is a Riemann sum $W_{0}$ on the same partition such that $\left|W-W_{0}\right|<\epsilon$.

Proof. Use the Vitali Covering Theorem to find finitely many intervals $I_{1}, I_{2}$, $\ldots, I_{k}$ such that

$$
m\left([a, b] \backslash\left(\cup_{j=1}^{k} I_{j}\right)\right)<\epsilon
$$

where $m$ is Lebesgue measure, and such that either

$$
|f(g(u))-f(g(v))|<\epsilon \quad \text { or } \quad|D g(u)-D g(v)|<\epsilon
$$

when $u$ and $v$ lie in an interval concentric with any $I_{j}$ and having twice the length of $I_{j}(1 \leq j \leq k)$.

Now $f$ and $D g$ are bounded. Choose $M$ with $M>|f|$ and $M>|D g|$. Let

$$
\delta=\frac{1}{2} \min _{j=1}^{k}\left(\text { length } I_{j}\right)
$$


and let the partition $a=x_{0}<x_{1}<\ldots<x_{n}=b$ have norm $<\delta$. Let

$$
W=\sum_{i=1}^{n} f\left(g\left(s_{i}\right)\right) D g\left(t_{i}\right)\left(x_{i}-x_{i-1}\right) \quad\left(s_{i}, t_{i} \in\left[x_{i-1}, x_{i}\right]\right) .
$$

Now

$$
\begin{aligned}
& \left|\sum^{*} f\left(g\left(s_{i}\right)\right) D g\left(t_{i}\right)\left(x_{i}-x_{i-1}\right)\right| \leq M^{2} \sum^{*}\left(x_{i}-x_{i-1}\right)<M^{*} \epsilon, \\
& \left|\sum^{*} f\left(g\left(t_{i}\right)\right) D g\left(t_{i}\right)\left(x_{i}-x_{i-1}\right)\right| \leq M^{2} \sum^{*}\left(x_{i}-x_{i-1}\right)<M^{*} \epsilon,
\end{aligned}
$$

where $\sum^{*}$ means sum on those indices $i$ for which $\left[x_{i-1}, x_{i}\right]$ does not meet any $I_{j}$. But if $\left[x_{i-1}, x_{i}\right]$ does meet some $I_{j}$, either

$$
\begin{aligned}
& \left|f\left(g\left(s_{i}\right)\right) D g\left(t_{i}\right)\left(x_{i}-x_{i-1}\right)-f\left(g\left(t_{i}\right)\right) D g\left(t_{i}\right)\left(x_{i}-x_{i-1}\right)\right| \leq M\left(x_{i}-x_{i-1}\right) \epsilon, \text { or } \\
& \left|f\left(g\left(s_{i}\right)\right) D g\left(t_{i}\right)\left(x_{i}-x_{i-1}\right)-f\left(g\left(s_{i}\right)\right) D g\left(s_{i}\right)\left(x_{i}-x_{i-1}\right)\right| \leq M\left(x_{i}-x_{i-1}\right) \epsilon .
\end{aligned}
$$

We use (1) and (2) to select $r_{i}=s_{i}$ or $t_{i}$ in such a way that if

$$
W_{0}=\sum_{j=1}^{n} f\left(g\left(r_{i}\right)\right) D g\left(r_{i}\right)\left(x_{i}-x_{i-1}\right),
$$

then

$$
\left|W-W_{0}\right|<2 M^{2} \epsilon+M \epsilon \sum_{i}\left(x_{i}-x_{i-1}\right)<2 M^{2} \epsilon+M \epsilon(b-a) .
$$

We leave the rest.

Proof. [Proof of Theorem 1] We deduce the first inequality from Lemmas 4 and 5. The last inequality is proved similarly by reversing appropriate inequalities in Lemma 4 and its proof. We leave the rest.

Proof. [Proof of Corollary 4] Let $a \leq u<v \leq b$ and let (**) hold. Let $g(u)=g(v)$. We define a function $h$ on $[a+v-u, b]$ as follows: put $h(x)=$ $g(x-v+u)$ for $a+v-u \leq x \leq v$ and $h(x)=g(x)$ for $v<x \leq b$. Note that $h(b)=g(b), h(v)=g(u)=g(v), h(a+v-u)=g(a)$,

$$
\overline{\int_{a+v-u}^{v}} f(h(t)) D h(t) d t=\overline{\int_{a}^{u}} f(g(t)) D g(t) d t,
$$




$$
\overline{\int_{v}^{b}} f(h(t)) D h(t) d t=\overline{\int_{v}^{b}} f(g(t)) D g(t) d t .
$$

By hypothesis,

$$
\begin{aligned}
\overline{\int_{a}^{u}} f(g(t)) D g(t) d t+ & \overline{\int_{u}^{v}} f(g(t)) D g(t) d t+\overline{\int_{v}^{b}} f(g(t)) D g(t) d t \\
= & \overline{\int_{g(a)}^{g(b)}} f(x) d x .
\end{aligned}
$$

By Theorem 1,

$$
\overline{\int_{a+v-u}^{v}} f(h(t)) D h(t) d t+\overline{\int_{v}^{b}} f(h(t)) D h(t) d t \geq \overline{\int_{h(a+v-u)}^{h(b)}} f(x) d x .
$$

Take the difference of the last two (in)equalities and obtain

$$
\overline{\int_{u}^{v}} f(g(t)) D g(t) d t \leq 0
$$

Similar arguments on the lower integrals give $\int_{u}^{v} f(g(t)) D g(t) d t \geq 0$. It follows that $f(g(t)) D g(t)$ is Riemann integrable on $[u, v]$.

We conclude this paper with some examples.

Example 1. Let $g(x)=|x|$ for $-1 \leq x \leq 1$, let $f(x)=1$ if $x$ is rational, and $f(x)=0$ if $x$ is irrational. Then $g(-1)=g(1)=1$, and

$$
\overline{\int_{g(-1)}^{g(1)}} f(x) d x=0<1=\overline{\int_{-1}^{1}} f(g(t)) D g(t) d t,
$$

So the equations in $(* *)$ need not hold in Theorem 1 when $g$ is not monotone.

Example 2. Let $E$ be a measurable subset of $[0,1]$ such that any subinterval of $[0,1]$ meets $E$ in a set of positive measure, and meets its complement $\complement E$ in a set of positive measure. let $g$ be the indefinite integral of the characteristic function of $E: g(x)=\int_{0}^{x} \chi_{E}(t) d t$. Then $g$ is strictly increasing, and the sets $A=\left\{t: g^{\prime}(t)=1\right\}$ and $B=\left\{t: g^{\prime}(t)=0\right\}$ are dense in $[0,1]$; hence $g(A)$ and $g(B)$ are dense in $g[0,1]$. Now let $f$ be identically 1 . It follows that

$$
\overline{\int_{0}^{1}} f(g(t)) D g(t) d t=1>m(E)=g(1)-g(0)=\overline{\int_{g(0)}^{g(1)}} f(x) d x .
$$

So the equations in $(* *)$ need not hold in Theorem 1 when $D g$ is not Riemann integrable. 
Example 3. Let $E$ and $g$ be as in Example 2, and put $k=g$. On the range of $k$ put $h(x)=1$ if $x \in k(B)$ and $h(x)=0$ if $x \notin k(B)$. Then $h(k(t)) D k(t)=0$ for all $t$, and

$$
\overline{\int_{0}^{1}} h(k(t)) D k(t) d t=0<m(E)=k(1)-k(0)=\overline{\int_{k(0)}^{k(1)}} h(x) d x .
$$

Of course the continuity hypothesis of the paper is not satisfied by $h$ and $D k$.

\section{References}

[F] J. Foran, A chain rule for the approximate derivatives and change of variables for the (D)-integral, Real Analysis Exchange 8 (1982-1983), $443-454$.

[G1] G. S. Goodman, Integration by substitution, Proc. Amer. Math. Soc. 70 (1978), 89-91.

[G2] G. S. Goodman, N-functions and integration by substitution, Rend. Sem. Mat. Fis. Milano 47 (1978), 123-134.

$[\mathrm{H}] \quad$ E. W. Hobson, The theory of functions of a real variable and the theory of Fourier series, vol. I, 3 ed., Harren Press, Washington D. C., 1950 (Section 360, pp. 506-507).

$[\mathrm{K}] \quad \mathrm{K}$. Krzyzewski, On change of variable in the Denjoy-Perron integral (I), (II), Coll. Math. 9 (1962), 99-104 and 317-323.

[S] S. Saks, Theory of the integral, 2nd. rev. ed., Dover, New York, 1964 (Dini's Theorem, p. 204).

[V] D. N. Sarkhel and R. Výborný, A change of variables theorem for the Riemann integral, Real Analysis Exchange 22 (1996-1997), no. 1, 390395.

[T] E. J. Townsend, Functions of a real variable, Henry Holt \& Company, New York, 1928 (Theorem XII, pp. 222-223). 